\title{
Research Article \\ Removal of Noise Oscillation Term Appearing in the Nonlinear Equation Solution
}

\author{
Yasir Khan, ${ }^{1}$ Hector Vázquez-Leal, ${ }^{2}$ \\ and Luis Hernandez-Martínez ${ }^{3}$ \\ ${ }^{1}$ Department of Mathematics, Zhejiang University, Hangzhou 310027, China \\ ${ }^{2}$ School of Electronic Instrumentation, University of Veracruz, Xalapa, VER, Mexico \\ ${ }^{3}$ Electronics Department, National Institute for Astrophysics, Optics and Electronics, \\ 72000 Tonantzintla, PUE, Mexico
}

Correspondence should be addressed to Yasir Khan, yasirmath@yahoo.com and Hector VázquezLeal, hvazquez@uv.mx

Received 27 April 2012; Accepted 4 July 2012

Academic Editor: J. Biazar

Copyright (C) 2012 Yasir Khan et al. This is an open access article distributed under the Creative Commons Attribution License, which permits unrestricted use, distribution, and reproduction in any medium, provided the original work is properly cited.

This paper suggests a novel modified Laplace method for removal of noise oscillation term appearing in the nonlinear equation solutions. The modified method overcomes the noise oscillation during the iteration procedure by suitable choice of an initial solution. Several examples are tested, and the obtained results suggest that this newly developed technique could lead to a promising tool and powerful improvement for many applications in differential and integral equations.

\section{Introduction}

Nonlinear science emerged in its present form following a series of decisive analytic, numerical, and experimental development took place in close interaction in the last three decades. And the application of many nonlinear science partial differential equations has attracted a great deal of attention in recent years. For example, partial differential equations are increasing used to model many problems in biology, chemistry, economic, engineering, physics, and other areas of applications. Many analytical and numerical techniques have been developed by various scientists to cope with the nonlinearity of such problems. In reality, the Laplace transform is one of only few methods that can be useful to linear systems with periodic or discontinuous driving inputs. In spite of its great usefulness in solving linear problems, however, the Laplace transform is totally incapable of handling nonlinear equations because of the difficulties that are caused by the nonlinear terms. Various 
ways have been proposed recently to deal with these nonlinearities such as the Adomian decomposition method [1-3], the Laplace decomposition method [4, 5], the homotopy perturbation method [6-14], variational approach [15, 16], Hamiltonian approach [17, 18], Boubaker polynomials expansion scheme $[19,20]$, and recently the homotopy perturbation transform method was proposed by Khan and $\mathrm{Wu}$ [21].

In this paper, we proposed a new method to solve any kind of nonlinear equations. This method is called modified homotopy perturbation transform method (MHPTM). It is important to note that the modified technique works effectively independent of other phenomena in some cases, or it may be combined with the powerful phenomena of the self-canceling "noise terms". We aim to extend the works of [21] and make further progress beyond the achievements made so far in this regard. The proposed modification will accelerate the rapid convergence of the series solution if compared with the standard homotopy perturbation transform method (HPTM), and therefore provides a major progress. The modified technique has been shown to be computationally efficient in several examples that are important to researchers in applied fields. In addition, the modified technique may give the exact solution for nonlinear equations without any need of the so-called Adomian polynomials. Although the modified technique needs only a slight variation from the standard homotopy perturbation transform method [21], the results are promising in that it minimizes the size of calculations needed. While this slight variation is rather simple, it does demonstrate the reliability and the power of the proposed modification. The modified homotopy perturbation transform method is much easier to implement as compared with the Adomian decomposition method and the Laplace decomposition method, where huge complexities are involved. Several examples are given to reconfirm the efficiency and accuracy of the proposed modified homotopy perturbation transform method.

\section{Description of the Method}

To illustrate the basic idea of this method, we consider a general nonlinear nonhomogeneous partial differential equation with initial condition of the following form:

$$
\begin{gathered}
D u(x, t)+R u(x, t)+N u(x, t)=g(x, t), \\
u(x, 0)=h(x), \quad u_{t}(x, 0)=f(x),
\end{gathered}
$$

where $f(x), h(x) \in C(R), D$ is the second order linear differential operator $D=\partial^{2} / \partial t^{2}, R$ the linear differential operator of less order than $D, N$ representing the general nonlinear differential operator, and $g(x, t)$ as the source term.

According to the homotopy perturbation transform method [21], we apply the Laplace transform (denoted throughout this paper by $L$ ) on both sides of (2.1) as follows:

$$
L[D u(x, t)]+L[R u(x, t)]+L[N u(x, t)]=L[g(x, t)]
$$


Journal of Applied Mathematics

Using the differentiation property of Laplace transform, we have

$$
\begin{gathered}
s^{2} L[u(x, t)]-s u(x, 0)-u_{t}(x, 0)+L[R u(x, t)]+L[N u(x, t)]=L[g(x, t)], \\
s^{2} L[u(x, t)]-s h(x)-f(x)+L[R u(x, t)]+L[N u(x, t)]=L[g(x, t)], \\
L[u(x, t)]=\frac{h(x)}{s}+\frac{f(x)}{s^{2}}+\frac{1}{s^{2}} L[g(x, t)]-\frac{1}{s^{2}} L[R u(x, t)+N u(x, t)] .
\end{gathered}
$$

Operating with Laplace inverse on both sides of (2.4) gives

$$
u(x, t)=G(x, t)-L^{-1}\left[\frac{1}{s^{2}} L[R u(x, t)+N u(x, t)]\right]
$$

where $G(x, t)$ represents the term arising from the source term and prescribed initial condition. The homotopy perturbation transform method [21] admits a solution in the form

$$
u(x, t)=\sum_{n=0}^{\infty} p^{n} u_{n}(x, t)
$$

The nonlinear term is decomposed as

$$
N u(x, t)=\sum_{n=0}^{\infty} p^{n} H_{n}(u)
$$

where $H_{n}$ are He's polynomials $[22,23]$ of $u_{0}, u_{1}, u_{2}, u_{3}, \ldots, u_{n}$ which can be calculated by the following formula:

$$
H_{n}=\frac{1}{n !} \frac{d^{n}}{d p^{n}}\left[N\left(\sum_{i=0}^{\infty} p^{i} u_{i}\right)\right]_{p=0}, \quad n=0,1,2,3, \ldots
$$

Using (2.7) and (2.6) in (2.5), we get

$$
\sum_{n=0}^{\infty} p^{n} u_{n}(x, t)=G(x, t)-p\left(L^{-1}\left[\frac{1}{s^{2}} L\left[R \sum_{n=0}^{\infty} p^{n} u_{n}(x, t)+\sum_{n=0}^{\infty} p^{n} H_{n}(u)\right]\right]\right)
$$


Comparing the coefficient of like powers of $p$, the following approximations are obtained:

$$
\begin{aligned}
& p^{0}: u_{0}(x, t)=G(x, t), \\
& p^{1}: u_{1}(x, t)=-L^{-1}\left[\frac{1}{s^{2}} L\left[R u_{0}(x, t)+H_{0}(u)\right]\right], \\
& p^{2}: u_{2}(x, t)=-L^{-1}\left[\frac{1}{s^{2}} L\left[R u_{1}(x, t)+H_{1}(u)\right]\right], \\
& p^{3}: u_{3}(x, t)=-L^{-1}\left[\frac{1}{s^{2}} L\left[R u_{2}(x, t)+H_{2}(u)\right]\right]
\end{aligned}
$$

Clearly, the general recursive relation is given by

$$
p^{n}: u_{n+1}(x, t)=-L^{-1}\left[\frac{1}{s^{2}} L\left[R u_{n}(x, t)+H_{n}(u)\right]\right], \quad n \geq 0 .
$$

The initial solution is important, and the choice of (2.10) as the initial solution always leads to noise oscillation/term during the iteration procedure. It is important to note that these terms may appear for inhomogeneous problems, whereas homogeneous problems do not generate noise terms. A necessary condition for the generation of the noise terms for inhomogeneous problems is that the zeroth component $u_{0}$ must contain the exact solution $u$ among other terms. The noise terms are defined as the identical terms, with opposite signs, that may appear in various components $u_{n}, n \geq 1$. It was formally shown that by canceling the noise terms that appear in $u_{0}$ and $u_{1}$ from $u_{0}$, even though $u_{1}$ contains further terms, the remaining noncancelled terms of $u_{0}$ may give the exact solution of the inhomogeneous problem. In order to overcome this shortcoming, we decompose $G(x, t)$ into two parts as

$$
G(x, t)=G_{0}(x, t)+G_{1}(x, t) .
$$

Instead of the iteration procedure, (2.10), (2.11), and (2.12), we suggest the following modification:

$$
\begin{aligned}
& p^{0}: u_{0}(x, t)=G_{0}(x, t) \\
& p^{1}: u_{1}(x, t)=G_{1}(x, t)-L^{-1}\left[\frac{1}{s^{2}} L\left[R u_{0}(x, t)+H_{0}(u)\right]\right] \\
& p^{2}: u_{2}(x, t)=-L^{-1}\left[\frac{1}{s^{2}} L\left[R u_{1}(x, t)+H_{1}(u)\right]\right] \\
& p^{3}: u_{3}(x, t)=-L^{-1}\left[\frac{1}{s^{2}} L\left[R u_{2}(x, t)+H_{2}(u)\right]\right]
\end{aligned}
$$


The solution through the modified homotopy perturbation transform method highly depends upon the choice of $G_{0}(x, t)$ and $G_{1}(x, t)$. We will show how to suitably choose $G_{0}(x, t)$ and $G_{1}(x, t)$ through the use of examples.

\section{Nonlinear Klein-Gordan Equations}

In order to elucidate the solution procedure of the modified method and how to choose a suitable initial solution, we consider first the nonlinear Klein-Gordon equations.

Example 3.1. Consider the following nonlinear Klein-Gordon equation:

$$
\begin{gathered}
u_{t t}(x, t)-u_{x x}(x, t)+u^{2}(x, t)=x^{2} t^{2}, \\
u(x, 0)=0, \quad u_{t}(x, 0)=x .
\end{gathered}
$$

Taking Laplace transform of both sides of (3.1) gives

$$
s^{2} u(x, s)-s u(x, 0)-u_{t}(x, 0)=\frac{2 ! x^{2}}{s^{3}}+L\left[u_{x x}-u^{2}\right]
$$

The initial conditions (3.2) imply

$$
u(x, s)=\frac{x}{s^{2}}+\frac{2 ! x^{2}}{s^{5}}+\frac{1}{s^{2}} L\left[u_{x x}-u^{2}\right] .
$$

The inverse Laplace transform applied to (3.4) results in

$$
u(x, t)=x t+\frac{x^{2} t^{4}}{12}+L^{-1}\left[\frac{1}{s^{2}} L\left[u_{x x}-u^{2}\right]\right] .
$$

Applying the homotopy perturbation transform method [21], we get

$$
\sum_{n=0}^{\infty} p^{n} u_{n}(x, t)=x t+\frac{x^{2} t^{4}}{12}+p\left(L^{-1}\left[\frac{1}{s^{2}} L\left[\sum_{n=0}^{\infty} p^{n} u_{n x x}(x, t)-\sum_{n=0}^{\infty} p^{n} H_{n}(u)\right]\right]\right)
$$


where $H_{n}(u)$ are He's polynomials $[22,23]$ that represent the nonlinear terms. In view of (3.6), recursive relation is given as follows:

$$
\begin{aligned}
p^{0}: u_{0}(x, t) & =x t+\frac{x^{2} t^{4}}{12} \\
p^{1}: u_{1}(x, t) & =L^{-1}\left[\frac{1}{s^{2}} L\left[u_{0 x x}-H_{0}(u)\right]\right] \\
& =L^{-1}\left[\frac{1}{s^{2}} L\left[u_{0 x x}-u_{0}^{2}\right]\right] \\
& =-\frac{x^{2} t^{4}}{12}-\frac{x^{4} t^{10}}{1080}-\frac{x^{3} t^{7}}{252}+\frac{t^{6}}{180} \\
p^{2}: u_{2}(x, t) & =L^{-1}\left[\frac{1}{s^{2}} L\left[u_{1 x x}-H_{1}(u)\right]\right] \\
& =L^{-1}\left[\frac{1}{s^{2}} L\left[u_{1 x x}-2 u_{0} u_{1}\right]\right] \\
& =-\frac{t^{6}}{180}+\frac{x^{3} t^{7}}{252}-\frac{11 x^{4} t^{10}}{45360}-\frac{x^{2} t^{12}}{71280}+\frac{37 x^{5} t^{13}}{7076160}+\frac{x^{6} t^{16}}{18662400}
\end{aligned}
$$

It is important to recall here that the noise terms appear between the two adjacent components, for example, $x^{2} t^{4} / 12$ in $u_{0}(x, t)$ and $u_{1}(x, t)$. The noise terms between the adjacent components can be cancelled using our modified version. The exact solution is

$$
u(x, t)=\sum_{n=0}^{\infty} u_{n}(x, t)=x t
$$

According to the modified homotopy perturbation transform method, we first set

$$
G(x, t)=x t+\frac{x^{2} t^{4}}{12}
$$

As suggested before, we split $G(x, t)$ into two parts as

$$
G_{0}(x, t)=x t, \quad G_{1}(x, t)=\frac{x^{2} t^{4}}{12} .
$$


According to the iteration algorithm equation (2.14), we obtain

$$
\begin{aligned}
p^{0}: u_{0}(x, t) & =x t \\
p^{1}: u_{1}(x, t) & =\frac{x^{2} t^{4}}{12}+L^{-1}\left[\frac{1}{s^{2}} L\left[u_{0 x x}-H_{0}(u)\right]\right] \\
& =\frac{x^{2} t^{4}}{12}+L^{-1}\left[\frac{1}{s^{2}} L\left[u_{0 x x}-u_{0}^{2}\right]\right] \\
& =\frac{x^{2} t^{4}}{12}-\frac{x^{2} t^{4}}{12}=0 \\
p^{i}: u_{i}(x, t) & =0, \quad i=2,3,4, \ldots
\end{aligned}
$$

One iteration leads to the exact solution $u(x, t)=x t$. It is obvious from this example that "noise terms" appeared in the standard homotopy perturbation transform method can be completely eliminated in our solution procedure.

Example 3.2. Consider the following nonlinear Klein-Gordon equation:

$$
\begin{gathered}
u_{t t}(x, t)-u_{x x}(x, t)+u^{2}(x, t)=2 x^{2}-2 t^{2}+x^{4} t^{4}, \\
u(x, 0)=0, \quad u_{t}(x, 0)=0 .
\end{gathered}
$$

By applying the aforesaid method subject to the initial conditions, we have

$$
u(x, s)=x^{2} \frac{2 !}{s^{3}}-\frac{4}{s^{5}}+x^{4} \frac{4 !}{s^{7}}+\frac{1}{s^{2}} L\left[u_{x x}-u^{2}\right] .
$$

The inverse of Laplace transform implies that

$$
u(x, t)=x^{2} t^{2}-\frac{t^{4}}{6}+x^{4} \frac{t^{6}}{30}+L^{-1}\left[\frac{1}{s^{2}} L\left[u_{x x}-u^{2}\right]\right]
$$

In view of the homotopy perturbation transform method [21], we have

$$
\begin{aligned}
\sum_{n=0}^{\infty} p^{n} u_{n}(x, t)= & x^{2} t^{2}-\frac{t^{4}}{6}+x^{4} \frac{t^{6}}{30} \\
& +p\left(L^{-1}\left[\frac{1}{s^{2}} L\left[\sum_{n=0}^{\infty} p^{n} u_{n x x}(x, t)-\sum_{n=0}^{\infty} p^{n} H_{n}(u)\right]\right]\right),
\end{aligned}
$$


where the nonlinear operator is decomposed as in (2.8) in terms of the He's polynomials. Matching both sides of (3.15), the components of $u$ can be defined as follows:

$$
\begin{gathered}
p^{0}: u_{0}(x, t)=x^{2} t^{2} \\
p^{1}: u_{1}(x, t)=-\frac{t^{4}}{6}+x^{4} \frac{t^{6}}{30}+L^{-1}\left[\frac{1}{s^{2}} L\left[u_{0 x x}-H_{0}(u)\right]\right]=0 .
\end{gathered}
$$

The solution procedure can continue, and we find the remaining components are all zero. We, therefore, obtain a closed-form solution which is $u(x, t)=x^{2} t^{2}$.

\section{Conclusion}

This paper suggests an effective modification of the Laplace transform method. A suitable choice of $G_{0}(x, t)$ and $G_{1}(x, t)$ results in an extremely simple solution procedure. The modified version is also valid for other nonlinear equations, and this paper can be used as a standard paradigm for other applications.

\section{Acknowledgment}

The authors wish to express their cordial thanks to the anonymous referees for useful suggestions and comments.

\section{References}

[1] G. Adomian, Solving Frontier Problems of Physics: The Decomposition Method, Kluwer Academic, Boston, Mass, USA, 1994.

[2] E. Hetmaniok, D. Słota, R. Witua, and A. Zielonka, "Comparison of the Adomian decomposition method and the variational iteration method in solving the moving boundary problem," Computers and Mathematics with Applications, vol. 61, no. 8, pp. 1931-1934, 2011.

[3] J. Biazar, "Solution of the epidemic model by Adomian decomposition method," Applied Mathematics and Computation, vol. 173, no. 2, pp. 1101-1106, 2006.

[4] S. A. Khuri, "A laplace decomposition algorithm applied to a class of nonlinear differential equations," Journal of Applied Mathematics, vol. 1, no. 4, pp. 141-155, 2001.

[5] Y. Khan, "An effective modification of the laplace decomposition method for nonlinear equations," International Journal of Nonlinear Sciences and Numerical Simulation, vol. 10, pp. 1373-1376, 2009.

[6] J. H. He, "Homotopy perturbation method for solving boundary value problems," Physics Letters A, vol. 350, no. 1-2, pp. 87-88, 2006.

[7] L. Xu, "He's homotopy perturbation method for a boundary layer equation in unbounded domain," Computers and Mathematics with Applications, vol. 54, no. 7-8, pp. 1067-1070, 2007.

[8] H. Koçak, T. Öziş, and A. Yildirim, "Homotopy perturbation method for the nonlinear dispersive $\mathrm{K}(\mathrm{m}, \mathrm{n}, 1)$ equations with fractional time derivatives," International Journal of Numerical Methods for Heat and Fluid Flow, vol. 20, no. 2, pp. 174-185, 2010.

[9] A. R. Ghotbi, A. Barari, M. Omidvar, and G. Domairry, "Application of homotopy perturbation and variational iteration methods to SIR epidemic model," Journal of Mechanics in Medicine and Biology, vol. 11, no. 1, pp. 149-161, 2011.

[10] M. Turkyilmazoglu, "Convergence of the homotopy perturbation method," International Journal of Nonlinear Sciences and Numerical Simulation, vol. 12, pp. 9-14, 2011.

[11] E. Hetmaniok, I. Nowak, D. Slota, and R. Witula, "Application of the homotopy perturbation method for the solution of inverse heat conduction problem," International Communications in Heat and Mass Transfer, vol. 39, no. 1, pp. 30-35, 2011. 
[12] H. Koçak and A. Yıldırım, "An efficient algorithm for solving nonlinear age-structured population models by combining homotopy perturbation and Pade techniques," International Journal of Computer Mathematics, vol. 88, no. 3, pp. 491-500, 2011.

[13] S. S. Ganji, A. Barari, and D. D. Ganji, "Approximate analysis of two-massspring systems and buckling of a column," Computers and Mathematics with Applications, vol. 61, no. 4, pp. 1088-1095, 2011.

[14] J. Biazar and Behzad Ghanbari, "The homotopy perturbation method for solving neutral functionaldifferential equations with proportional delays," Journal of King Saud University, vol. 24, no. 4, pp. 33-37, 2012.

[15] Y. Khan, N. Faraz, and A. Yildirim, "New soliton solutions of the generalized Zakharov equations using He's variational approach," Applied Mathematics Letters, vol. 24, no. 6, pp. 965-968, 2011.

[16] L. Xu and N. Zhang, "A variational approach to analyzing catalytic reactions in short monoliths," Computers and Mathematics with Applications, vol. 58, no. 11-12, pp. 2460-2463, 2009.

[17] Y. Khan, Q. Wu, H. Askari, Z. Saadatnia, and M. Kalami-Yazdi, "Nonlinear vibration analysis of a rigid rod on a circular surface via hamiltonian approach," Mathematical and Computational Applications, vol. 15, no. 5, pp. 974-977, 2010.

[18] L. Xu, "A Hamiltonian approach for a plasma physics problem," Computers and Mathematics with Applications, vol. 61, no. 8, pp. 1909-1911, 2011.

[19] W. X. Yue, H. Koçak, D. H. Zhang, and A. Yildirim, “A second attempt to establish an analytical expression to steam-water dipole orientation parameter using the boubaker polynomials expansion scheme," Journal of Structural Chemistry, vol. 52, no. 1, pp. 106-110, 2011.

[20] H. Koçak, Z. Dahong, and A. Yildirim, "A range-free method to determine Antoine vapor-pressure heat transfer-related equation coefficients using the Boubaker polynomial expansion scheme," Russian Journal of Physical Chemistry A, vol. 85, no. 5, pp. 900-902, 2011.

[21] Y. Khan and Q. Wu, "Homotopy perturbation transform method for nonlinear equations using He's polynomials," Computers and Mathematics with Applications, vol. 61, no. 8, pp. 1963-1967, 2011.

[22] Y. Khan, M. Madani, A. Yıldırım, M. A. Abdou, and N. Faraz, "A new approach to Van der Pol's oscillator problem," Zeitschrift für Naturforschung, vol. 66, pp. 620-624, 2011.

[23] A. Ghorbani, "Beyond Adomian polynomials: He polynomials," Chaos, Solitons and Fractals, vol. 39, no. 3, pp. 1486-1492, 2009. 


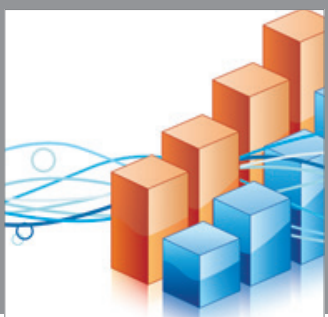

Advances in

Operations Research

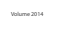

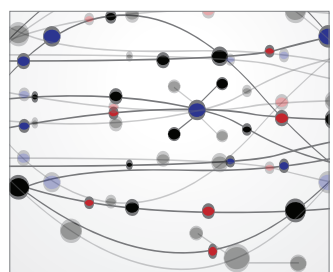

\section{The Scientific} World Journal
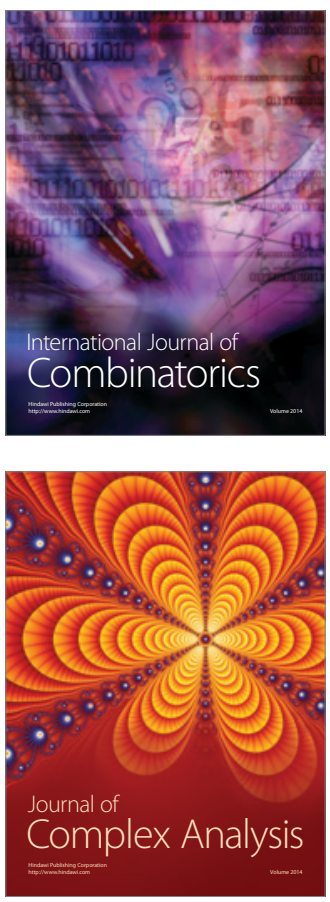

International Journal of

Mathematics and

Mathematical

Sciences
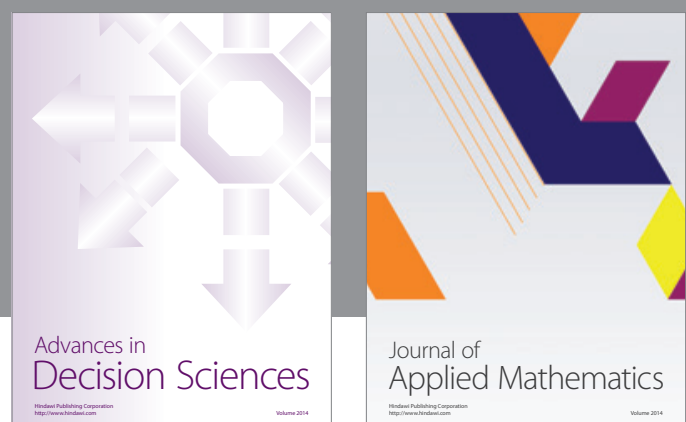

Journal of

Applied Mathematics
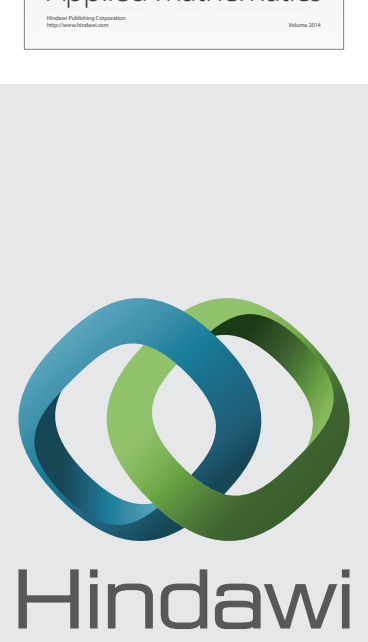

Submit your manuscripts at http://www.hindawi.com
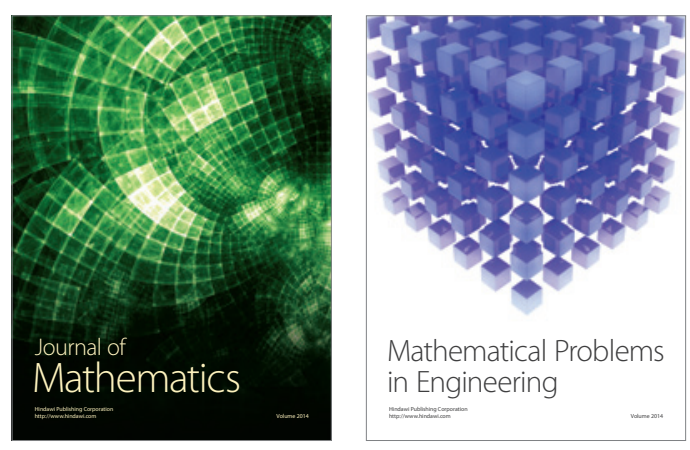

Mathematical Problems in Engineering
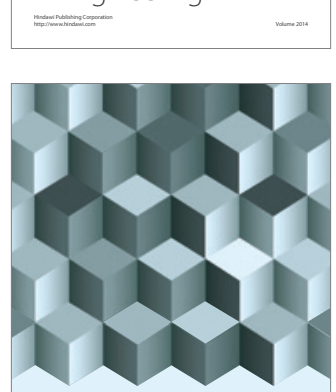

Journal of

Function Spaces
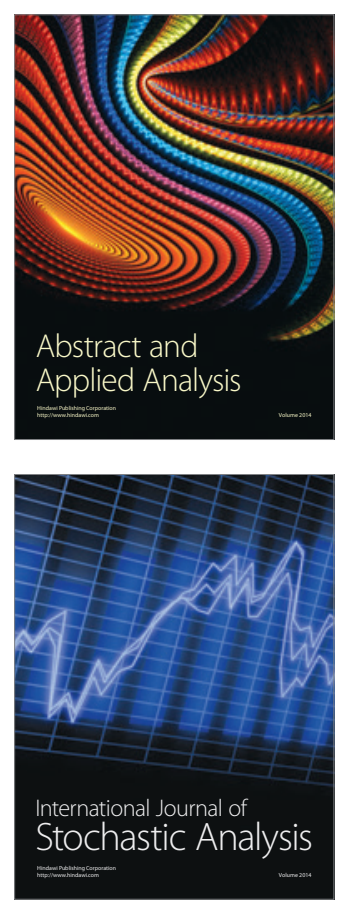

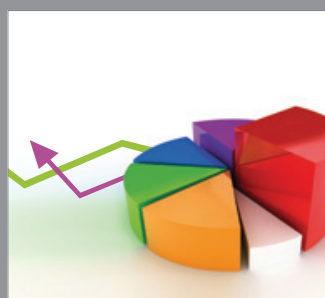

ournal of

Probability and Statistics

Promensencen
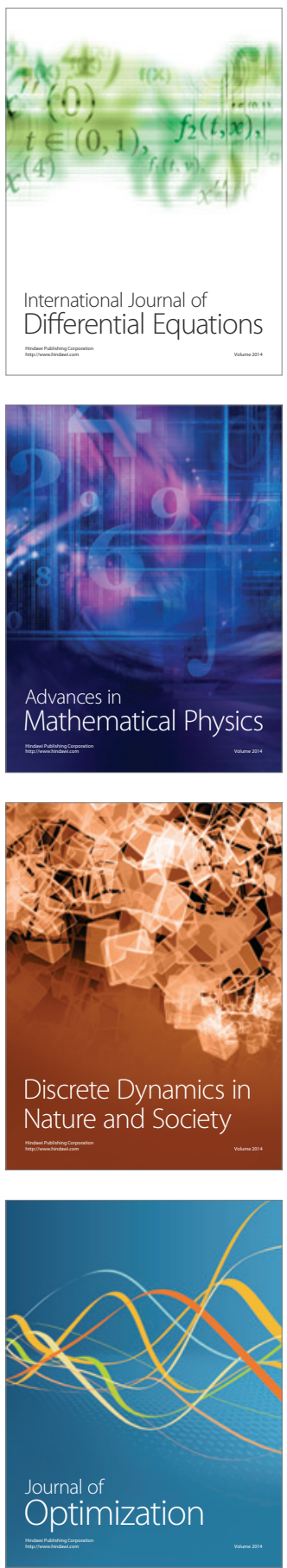